JAMA | US Preventive Services Task Force | RECOMMENDATION STATEMENT

Screening for Cognitive Impairment in Older Adults US Preventive Services Task Force Recommendation Statement

US Preventive Services Task Force

IMPORTANCE Dementia (also known as major neurocognitive disorder) is defined by a significant decline in 1 or more cognitive domains that interferes with a person's independence in daily activities. Dementia affects an estimated 2.4 to 5.5 million individuals in the United States, and its prevalence increases with age.

OBJECTIVE To update its 2014 recommendation, the US Preventive Services Task Force (USPSTF) commissioned a review of the evidence on screening for cognitive impairment, including mild cognitive impairment and mild to moderate dementia, in community-dwelling adults, including those 65 years or older residing in independent living facilities.

POPULATION This recommendation applies to community-dwelling older adults 65 years or older, without recognized signs or symptoms of cognitive impairment.

EVIDENCE ASSESSMENT The USPSTF concludes that the evidence is lacking, and the balance of benefits and harms of screening for cognitive impairment cannot be determined.

RECOMMENDATION The USPSTF concludes that the current evidence is insufficient to assess the balance of benefits and harms of screening for cognitive impairment in older adults. (I statement)

JAMA. 2020;323(8):757-763. doi:10.1001/jama.2020.0435
Editorial page 722

Related article page 764 and JAMA Patient Page page 800

$+$

Audio and Supplemental content

CME Quiz at

jamacmelookup.com and CME Questions page 786

$+$

Related article at jamainternalmedicine.com

Author/Group Information: The US Preventive Services Task Force (USPSTF) members appear at the end of this article.

Corresponding Author: Douglas $\mathrm{K}$ Owens, MD, MS, Stanford University, 615 Crothers Way, Encina Commons, Mail Code 6019, Stanford, CA 94305-6006 (chair@uspstf.net).

\title{
Summary of Recommendation
}

The USPSTF concludes that the current evidence is insufficient to assess the balance of benefits and harms of screening for cognitive impairment in older adults.

See the Figure for a more detailed summary of the recommendation for clinicians. See the Practice Considerations section for suggestions for practice regarding the I statement.

\section{Importance}

According to the American Psychiatric Association's Diagnostic and Statistical Manual of Mental Disorders (Fifth Edition) (DSM-5), dementia (also known as major neurocognitive disorder) is defined by a significant decline in 1 or more cognitive domains that interferes with a person's independence in daily activities. The 6 cognitive domains identified in the DSM-5 are complex attention, executive function, learning and memory, language, perceptual motor function, and social cognition. ${ }^{1}$ Dementia affects an estimated 2.4 to 5.5 million persons in the United States, and its prevalence increases with age. Dementia affects an estimated $3.2 \%$ of persons aged 65 to 74 years, $9.9 \%$ of those aged 75 to 84 years, and $29.3 \%$ of those 85 years or older. ${ }^{2}$

Mild cognitive impairment $(\mathrm{MCl})$ differs from dementia in that the impairment is not severe enough to interfere with independent daily functioning. Some persons with $\mathrm{MCl}$ may progress to dementia, while some do not. One systematic review found that $32 \%$ of persons with $\mathrm{MCl}$ develop dementia over 5 years. ${ }^{3}$ However, studies have also shown that between $10 \%$ and $40 \%$ of persons with $\mathrm{MCl}$ may return to normal cognition over approximately 4 to 5 years. ${ }^{4-6}$ The prevalence of $\mathrm{MCl}$ is difficult to estimate, in part because of differing diagnostic criteria, leading to a wide range of prevalence estimates (3\%-42\%) in adults 65 years or older. ${ }^{7,8}$ In this recommendation statement, "cognitive impairment" refers to both dementia and $\mathrm{MCl}$.

\section{USPSTF Assessment of Magnitude of Net Benefit}

The US Preventive Services Task Force (USPSTF) concludes that the evidence is lacking, and the balance of benefits and harms of screening for cognitive impairment cannot be determined (Figure and Table). 


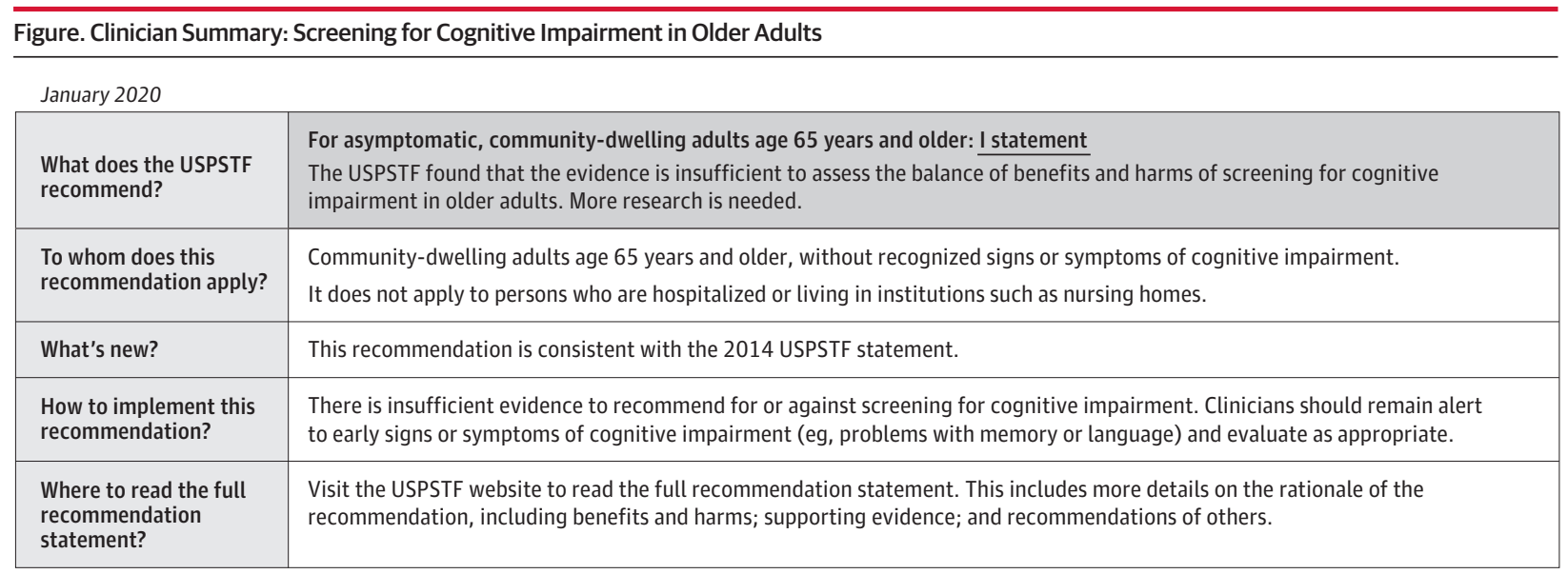

The USPSTF recognizes that clinical decisions involve more considerations than evidence alone. Clinicians should understand the evidence but individualize decision-making to the specific patient or situation.

USPSTF indicates US Preventive Services Task Force.

\begin{tabular}{|c|c|}
\hline \multicolumn{2}{|c|}{ Table. Summary of USPSTF Rationale ${ }^{a}$} \\
\hline Rationale & Assessment \\
\hline Detection & $\begin{array}{l}\text { - Adequate evidence that some screening tools have relatively high sensitivity and specificity for the detection of dementia } \\
\text { - When the prevalence of dementia is high (eg, in persons } \geq 85 \mathrm{y} \text { ), positive predictive values can be greater than } 50 \% \text {. However, } \\
\text { because of lower prevalence, the positive predictive value can be closer to } 20 \% \text { in unselected populations of adults aged } 65 \text { to } 74 \mathrm{y} \\
\text { - Sensitivity and specificity of screening tools is generally lower for the detection of } \mathrm{MCl} \text { than it is for dementia }\end{array}$ \\
\hline $\begin{array}{l}\text { Benefits of early detection } \\
\text { and intervention and } \\
\text { treatment }\end{array}$ & $\begin{array}{l}\text { - Inadequate direct evidence on the benefits of screening for cognitive impairment } \\
\text { - Adequate evidence that AChEIs and memantine have a small effect on measures of cognitive function in the short term for patients } \\
\text { with mild to moderate dementia, but it is uncertain if the effects reported in studies are clinically meaningful or sustained } \\
\text { over the long term } \\
\text { - Inadequate evidence on the benefits of other medications or supplements (eg, statins, antihypertensives, or vitamins) and } \\
\text { nonpharmacologic interventions targeted to patients } \\
\text { - Adequate evidence that interventions to support caregivers have a small effect on measures of caregiver burden and depression, } \\
\text { but the clinical importance of these effects are uncertain, and the generalizability of these findings to persons with previously } \\
\text { - Innrecognized dementia, detected by screening, is not known }\end{array}$ \\
\hline $\begin{array}{l}\text { Harms of early detection } \\
\text { and intervention and } \\
\text { treatment }\end{array}$ & $\begin{array}{l}\text { - Inadequate direct evidence on the harms of screening for cognitive impairment } \\
\text { - Inadequate evidence on the harms of nonpharmacologic interventions targeted at the patient, caregiver, or both } \\
\text { - Adequate evidence that AChEls are associated with adverse effects, which overall are small but occasionally serious, including } \\
\text { syncope or falls }\end{array}$ \\
\hline USPSTF assessment & - Evidence on screening for cognitive impairment is lacking and the balance of benefits and harms cannot be determined \\
\hline
\end{tabular}

\section{Practice Considerations}

\section{Patient Population Under Consideration}

This recommendation applies to community-dwelling older adults 65 years or older, without recognized signs or symptoms of cognitive impairment. Early detection and diagnosis of dementia through the assessment of signs and symptoms recognized by the patient, family, or clinician, which may be subtle, are not considered screening and are therefore not the focus of this recommendation.

\section{Assessment of Risk}

Increasing age is the strongest known risk factor for cognitive impairment. ${ }^{9}$ The $\varepsilon 4$ allele of the apolipoprotein $\mathrm{E}(\mathrm{apoE})$ gene is a reported risk factor for Alzheimer disease in white and Asian populations. ${ }^{10}$ Other risk factors include cardiovascular risk factors (eg, diabetes, hypertension, or hypercholesterolemia), depression, physical frailty, low education level, and low social support level. ${ }^{10-13}$ Several dietary and lifestyle factors have been reported as associated with decreased risk of cognitive impairment. These include adequate folic acid intake, low saturated fat intake, higher longer-chain omega-3 fatty acid intake, high fruit and vegetable intake, the Mediterranean diet, moderate alcohol intake (1 to 6 drinks per week vs abstention), higher educational attainment, cognitive engagement, social engagement, and higher physical activity levels. ${ }^{10,11,14,15}$

\section{Screening Tests}

Many different brief screening tests for cognitive impairment are available. Screening tests generally include asking patients to perform a series of tasks that assess 1 or more domains of cognitive function. These tests are not intended to diagnose $\mathrm{MCl}$ or dementia. A positive screening test result should lead to additional testing that can include blood tests, radiology examinations, and a medical and neuropsychologic evaluation to confirm the diagnosis of dementia and determine its subtype. 
The most well-studied screening instrument is the MiniMental State Examination (MMSE). Other screening instruments include the clock drawing test (CDT), Memory Impairment Screen (MIS)/MIS by Telephone (MIS-T), Mental Status Questionnaire (MSQ), Mini-Cog verbal fluency, 8-Item Informant Interview (AD8), Functional Activities Questionnaire (FAQ), 7-Minute Screen (7MS), Abbreviated Mental Test (AMT), Montreal Cognitive Assessment (MoCA), St. Louis University Mental Status Examination (SLUMS), Telephone Instrument for Cognitive Status (TICS), and Informant Questionnaire on Cognitive Decline in the Elderly (IQCODE). For more information on all screening instruments reviewed by the USPSTF, see the full evidence report. ${ }^{16,17}$

\section{Treatment and Interventions}

Treatment of cognitive impairment may focus on one or more signs and symptoms, including cognition, quality of life, mood, and behavioral impairments.

Several pharmacologic and nonpharmacologic interventions aim to slow, prevent, or reverse cognitive impairment; stabilize or improve functional performance; improve caregiver burden and depression; or improve patient, caregiver, and clinician decision-making. Pharmacologic treatments approved by the US Food and Drug Administration for Alzheimer disease include acetylcholinesterase inhibitors (AChEls) and memantine. Nonpharmacologic interventions include cognitive training, cognitive rehabilitation, cognitive stimulation interventions, exercise, peer support, psychoeducation, and care management.

\section{Suggestions for Practice Regarding the I Statement}

\section{Potential Preventable Burden}

Dementia affects an estimated 2.4 to 5.5 million persons in the United States, and its prevalence increases with age. Dementia affects an estimated $9.9 \%$ of persons aged 75 to 84 years and $29.3 \%$ of those 85 years or older. ${ }^{2}$ Subjective memory issues are common in adults, with studies showing that approximately $50 \%{ }^{18}$ to $75 \%{ }^{19}$ of adults have at least minor concerns about their memory.

Although there is insufficient evidence to recommend for or against screening for cognitive impairment, there may be important reasons to identify cognitive impairment early. Burdens of cognitive impairment include direct effects on the patient (eg, loss of function and relationships, financial misjudgments, and nonadherence with recommended therapies), direct effects on caregivers (eg, burden and depression), and effects on society (eg, costs of care). Early detection of cognitive impairment can allow for identification and treatment of reversible causes, may help clinicians anticipate problems patients may have in understanding and adhering to medical treatment plans, and may also be useful by providing a basis for advance planning on the part of patients and families. Clinicians should remain alert to early signs or symptoms of cognitive impairment (eg, problems with memory or language) and evaluate the individual as appropriate. However, none of the potential benefits of screening have been clearly demonstrated in clinical trials.

\section{Potential Harms}

Evidence about the harms of screening is limited. One potential harm is labeling a person with an illness that is typically progressive and for which treatment appears to have limited effectiveness. Some studies have shown higher stress, greater depression, and lower quality of life in persons aware of a diagnosis of cognitive impairment, ${ }^{20}$ while others have found no such association. ${ }^{21,22}$ Evidence about the effects of false-positive results is limited. AChEls are associated with harms, some of which are serious, including central nervous system disturbances, bradycardia, and falls. Evidence about the harms of nonpharmacologic interventions is limited, but these harms are assumed to be small.

\section{Current Practice}

Most commonly, evaluation for or diagnosis of cognitive impairment occurs as a result of patient- or caregiver-reported concerns or symptoms, or clinician's suspicion. As many as $29 \%$ to $76 \%$ of patients with dementia are unrecognized in the primary care setting. ${ }^{23-25}$ In 2011, Medicare added detection of cognitive impairment to its annual wellness visit. The Centers for Medicare \& Medicaid Services recommend assessing a patient's cognitive function by direct observation; considering information and concerns reported by the patient, family members, friends, caregivers, and others; and, if deemed appropriate, using a brief validated, structured cognitive assessment tool. ${ }^{26}$

\section{Additional Tools and Resources}

The National Institute on Aging has useful information on the definition, detection, and management of cognitive impairment for patients and clinicians, including links to some screening instruments, on its website. ${ }^{27}$

\section{Other Related USPSTF Recommendations}

The USPSTF has recommendations related to several risk factors for cognitive impairment, including pharmacotherapy and counseling for tobacco cessation ${ }^{28}$; screening and counseling to reduce unhealthy alcohol use ${ }^{29}$; counseling to promote healthful diet and physical activity ${ }^{30,31}$; statins to reduce cardiovascular disease risk ${ }^{32}$; and screening for hypertension, ${ }^{33}$ abnormal blood glucose levels, ${ }^{34}$ and depression. ${ }^{35}$

\section{Update of Previous USPSTF Recommendation}

This recommendation updates the 2014 USPSTF recommendation on screening for cognitive impairment in older adults. In 2014, the USPSTF concluded that the evidence was insufficient to assess the balance of benefits and harms of screening for cognitive impairment. ${ }^{36}$ For the current recommendation, the USPSTF again concludes that the evidence is insufficient to assess the balance of benefits and harms of screening for cognitive impairment in older adults.

\section{Supporting Evidence}

\section{Scope of Review}

To update its 2014 recommendation, the USPSTF commissioned a systematic review ${ }^{16,17}$ on the benefits and harms of screening for cognitive impairment, including $\mathrm{MCl}$ and mild to moderate dementia, in community-dwelling adults, including those 65 years or older 
residing in independent living facilities. The systematic review included evidence on the accuracy of screening tests to detect cognitive impairment and the benefits and harms of treatments and interventions for older adults with cognitive impairment and their caregivers. The review focused on older adults with $\mathrm{MCl}$ or mild to moderate dementia, as these are the patients most likely to be identified by screening.

\section{Accuracy of Screening Tests}

The USPSTF found 59 studies that evaluated 49 screening instruments to detect cognitive impairment. Among the studies, the prevalence of dementia ranged from $1 \%$ to $47 \%$ and the prevalence of $\mathrm{MCl}$ ranged from $10 \%$ to $52 \%$. The mean age of participants ranged from 68 to 95 years. Education level of participants, which was reported in 22 studies, ranged from a mean of 5 to 16 years of education. Most of the instruments evaluated were very brief (evaluation time $\leq 5$ minutes) or brief (evaluation time $\leq 10$ minutes). ${ }^{16,17}$

Screening instruments evaluated in more than 1 study included the MMSE, CDT, MIS/MIS-T, MSQ, Mini-Cog verbal fluency, AD8, FAQ, 7MS, AMT, Lawton Instrumental Activities of Daily Living Scale, MoCA, SLUMS, TICS, and IQCODE. The MMSE was the most evaluated instrument (30 studies). The MMSE is an 11-item instrument with a maximum score of 30 points. In a pooled analysis of 14 studies using cutoffs of 23 or less or 24 or less (score considered a positive screening result) $(n=11972)$, the MMSE had a sensitivity of $0.89(95 \% \mathrm{Cl}, 0.85$ to 0.92$)$ and a specificity of $0.90(95 \% \mathrm{Cl}, 0.86$ to 0.93$)$ to detect dementia. The other instruments were studied in fewer studies ( 2 to 9 studies), and cutoffs for screening instruments often varied across studies. Sensitivity and specificity of these instruments to detect dementia varied widely in these studies, from 0.43 to 1.0 and 0.54 to 1.0, respectively. Across all instruments, test accuracy (ie, sensitivity and specificity) was generally higher to detect dementia compared with $\mathrm{MCl}^{16,17}$

\section{Benefits of Early Detection and Treatment}

The USPSTF found 1 trial, IU-CHOICE ${ }^{37}(n=4005)$, that examined the effect of screening for cognitive impairment on health-related quality of life (HRQOL), health care utilization, and measures of advance care planning. This trial found no differences in HRQOL at 1,6 , or 12 months and no differences in health care utilization or measures of advance care planning at 12 months in screening compared with control groups. Limitations of this trial include that $38 \%$ of persons assessed for eligibility refused to participate, and $66 \%$ of participants who screened positive refused diagnostic assessment and follow-up. Additionally, the trial was missing $42 \%$ of HRQOL outcomes data at 12 months.

The USPSTF found 48 trials ( $n=22431$ ) that evaluated the effects of AChEls (donepezil [18 studies; $n=6209$ ], galantamine [10 studies; $n=7464$ ], rivastigmine [8 studies; $n=4569$ ], and memantine [12 studies; $n=4189$ ]) on global cognitive function. Many of these trials reported differences in scores on the Alzheimer Disease Assessment Scale-Cognitive Subscale (ADASCog-11) or the MMSE. AChEls and memantine improved measures of global cognitive function in the short term, but the magnitude of change was small. In pooled analyses, the differences in changes between patients taking AChEls or memantine compared with patients taking placebo ranged from approximately 1 to 2.5 points on the ADAS-Cog-11 (scale range, 0-70) and 0.5 to 1 points on the MMSE (scale range, $0-30$ ) over 3 months to 3 years of follow-up. ${ }^{16,17}$ Changes of this magnitude would generally not be considered clinically important. ${ }^{38}$ AChEls and memantine increased the likelihood of improving or maintaining patients' global function, typically as assessed by the Clinician's InterviewBased Impression of Change Plus Informant Input (CIBIC+), by $15 \%$ (for memantine) to $50 \%$ (for rivastigmine) in the short term; however, change at longer-term follow-up was not reported. ${ }^{16,17}$

Most of the evidence on the effectiveness of AChEls and memantine comes from trials involving persons with dementia, particularly those with moderate as opposed to mild dementia, and most commonly Alzheimer disease. Four trials ( $n=1919)$ tested donepezil or memantine in persons with $\mathrm{MCl}$. These trials showed no improvement in global cognitive function. ${ }^{16,17}$

Twenty-nine trials ( $n=6489$ ) evaluated other medications or supplements, including antihypertensives, statins, nonsteroidal anti-inflammatory drugs, gonadal steroids, and dietary supplements and vitamins (multivitamins, $B$ vitamins, vitamin $E$, and omega-3 fatty acids). None of the trials found any improvement in global cognition or physical function in persons with mild to moderate dementia or $\mathrm{MCl}^{16,17}$

Sixty-one trials ( $n=7847$ ) evaluated nonpharmacologic patient-level interventions, including cognitive-focused, exercise, multicomponent, and other interventions. In general, these trials were small and of limited duration. This body of evidence represented persons with dementia and $\mathrm{MCl}$, with a mean age of 75 years. Among all interventions, there was no clear improvement in global or domain-specific measures of cognitive function compared with controls at 3 months to 2 years of follow-up. ${ }^{16,17}$

Fifty-eight trials ( $n=9139$ ) evaluated psychoeducation interventions targeting the caregiver or caregiver-patient dyad. Most trials targeted patients with dementia, and the average MMSE score in studies that reported it was 16.2, consistent with moderate dementia. The interventions were highly variable, with most including training in problem solving, communications, and stress management, in addition to providing information about dementia and community resources. Overall, there was a small improvement in caregiver burden and depression measures, primarily in persons caring for patients with moderate dementia. For example, a pooled analysis of 9 trials $(n=1089)$ that reported change in the Zarit-22 (a 22-item scale of caregiver burden, with a score ranging from 0 to 88) found an average 2.5-point improvement (mean difference, -2.5 [95\% Cl, -3.9 to -1.0$]$ ), and a pooled analysis of 20 trials $(n=2603)$ that reported change in the Center for Epidemiologic Studies Depression Scale (CESD) (a 20-item scale of depressive symptoms, with a score ranging from 0 to 60 ) found a mean 2.67 point improvement (mean difference, $-2.67[95 \% \mathrm{Cl},-3.45$ to $-1.48]$ ) in the intervention groups. ${ }^{16,17}$

Seventeen trials ( $n=3039$ ) evaluated care or case management interventions. All interventions were intended for patients with dementia. Of the 12 trials that reported caregiver burden outcomes, 5 found a statistically significant improvement in scores. A pooled analysis of 8 trials $(n=1215)$ found a standardized pooled effect of $-0.54(95 \% \mathrm{Cl},-0.85$ to -0.22$)$, translating to a betweengroup difference of approximately 3.5 to 4 points on the Zarit-22. Seven trials reported caregiver depression outcomes. A pooled 
analysis of 4 of these trials $(n=668)$ showed no improvement in caregiver depression measures. ${ }^{16,17}$

Two trials reported the effects of interventions on planning or decision-making outcomes. One trial $(n=111)^{39}$ reported the effects of a psychoeducational intervention on caregivers' planning or decision-making related to caring for a relative with dementia. Three months after the intervention, caregivers in both the intervention and usual care groups reported significantly better planning for future needs, and there was no difference between the intervention and usual care groups. A second trial (the Maximizing Independence at Home [MIND] Care Coordination Trial) $(n=303)^{40}$ assessed whether a care coordination intervention reduced unmet care needs related to dementia compared with usual care. Evaluators assessed 19 common care needs for participants ( 71 items) and caregivers (15 items) as being either "fully met" or "unmet." After 18 months, there was no difference in reduction of total percentage of unmet needs in the intervention group vs usual care.

Overall, the body of evidence on the potential benefits of screening for cognitive impairment is limited by several factors. These include the short duration of most trials (often $\leq 6$ months for pharmacologic agents and $\leq 1$ year for nonpharmacologic interventions), as well as the heterogeneous nature of interventions and inconsistency in the outcomes reported, which make cross-study comparisons difficult. As noted, for interventions for which studies reported an improvement in measures, the average effect sizes were small and of uncertain clinical importance. In addition, no interventions specifically targeted a screen-detected population. Most of the evidence suggesting improvement is applicable to persons with moderate dementia; thus, its applicability to a screendetected population is uncertain.

\section{Harms of Screening and Treatment}

The USPSTF found 1 trial (IU-CHOICE) ${ }^{37}$ that addressed the potential harms of screening for cognitive impairment. This trial and its limitations are described above. The trial found no differences in depression or anxiety (as measured by PHQ-9 and GAD-7) in participants randomized to screening vs control groups at 1,6 , or 12 months.

Forty-eight randomized clinical trials ( $n=22431)$ and 3 observational studies ( $n=190076$ ) reported on the harms of treatment with AChEls and memantine. ${ }^{16,17}$ Adverse effects of medications were common. Adverse events were significantly higher in patients receiving AChEls, and more patients receiving AChEls withdrew from studies or discontinued their medication compared with patients receiving placebo. Memantine was better tolerated, with no increase in adverse events or withdrawal rates compared with placebo. Overall, there was no increase in serious adverse events in patients taking AChEls. However, some individual studies reported increased rates of serious adverse events, such as bradycardia, syncope, falls, and need for pacemaker placement among patients taking AChEls.

Twenty-one of the trials ( $n=5688)$ that evaluated other medications or supplements reported on harms. Harms were not clearly significantly increased in intervention groups compared with control groups. ${ }^{16,17}$

For nonpharmacologic patient-level interventions, few studies reported on harms. Little harm was evident in the 12 studies $(n=2370)$ that reported it. ${ }^{16,17}$
Response to Public Comment

A draft version of this recommendation statement was posted for public comment on the USPSTF website from September 10, 2019, to October 7, 2019. Several comments agreed that the evidence on screening for cognitive impairment is insufficient. Several comments expressed that readers might misinterpret the I statement as a recommendation against screening, or interpreted the I statement as a recommendation against screening. In response, the USPSTF wants to clarify that its I statement is a conclusion that the evidence is insufficient to assess the balance of benefits and harms of screening for cognitive impairment and is neither a recommendation for nor against screening. Several comments disagreed with the I statement and felt that the USPSTF should recommend screening for cognitive impairment based on the potential benefits mentioned in the Practice Considerations section. In response, the USPSTF clarified that while there may be important reasons to identify cognitive impairment early, none of the potential benefits mentioned in this section have been clearly demonstrated in controlled trials. Several comments noted that cognitive impairment often goes unrecognized. The USPSTF agrees and added language to the Practice Considerations section noting that clinicians should remain alert to early signs or symptoms of cognitive impairment. Some comments suggested additional studies that should be considered by the USPSTF. Some of the suggested studies investigated the prevention of cognitive impairment by controlling risk factors, such as hypertension, or by use of a multidomain intervention (eg, diet, physical activity, cognitive training, and risk factor control). However, none of these suggested studies met inclusion criteria for this recommendation; that is, they were not studies of screening for and/or treatment of cognitive impairment or mild to moderate dementia. Also, the USPSTF added the American Academy of Neurology's guidelines on the detection of cognitive impairment to the Recommendations of Others section.

How Does Evidence Fit With Biological Understanding?

Dementia can be the result of varied and different pathophysiologic processes affecting the brain, and the exact causal mechanism for many types of dementia is unknown. Therefore, the development of early interventions that result in important clinical effects on dementia has been challenging. The most common cause of dementia in the United States is Alzheimer disease, which is the target of all current drugs approved by the US Food and Drug Administration for dementia. Since current medical therapies for dementia do not appear to affect its long-term course, the potential benefit of screening may be in devising effective interventions that can help patients and caregivers prepare for managing the symptoms and consequences of dementia.

\section{Research Needs and Gaps}

- More research is needed on the effect of screening and early detection of cognitive impairment ( $\mathrm{MCl}$ and mild to moderate dementia) on important patient, caregiver, and societal outcomes, including decision-making, advance planning, and caregiver outcomes.

- The body of evidence on screening and interventions for cognitive impairment would benefit from more consistent definitions 
and reporting of outcomes to allow comparisons across trials, especially from trials with longer-term follow-up.

- Studies are needed of the effects of caregiver or patientcaregiver dyad interventions on delay or prevention of institutionalization, and the effects of delay in institutionalization on caregivers.

- Research is needed on treatments that clearly affect the longterm clinical course of cognitive impairment. It is also important that studies on screening and interventions for cognitive impairment report harms and reasons for attrition of trial participants.

\section{Recommendations of Others}

The American Academy of Family Physicians supports the 2014 USPSTF recommendation on screening for cognitive impairment in older adults. ${ }^{41}$ The American Academy of Neurology has published guidance on the detection of cognitive impairment during the annual wellness visit and recommends the use of a brief, validated cognitive assessment instrument to assess for cognitive impairment. ${ }^{42}$

\section{ARTICLE INFORMATION}

Accepted for Publication: January 16, 2020. The US Preventive Services Task Force (USPSTF) members: Douglas K. Owens, MD, MS; Karina W. Davidson, PhD, MASc; Alex H. Krist, MD, MPH; Michael J. Barry, MD; Michael Cabana, MD, MA, $\mathrm{MPH}$; Aaron B. Caughey, MD, PhD; Chyke A. Doubeni, MD, MPH; John W. Epling Jr, MD, MSEd; Martha Kubik, PhD, RN; C. Seth Landefeld, MD Carol M. Mangione, MD, MSPH; Lori Pbert, PhD; Michael Silverstein, MD, MPH; Melissa A. Simon, MD, MPH; Chien-Wen Tseng, MD, MPH, MSEE; John B. Wong, MD.

Affiliations of The US Preventive Services Task Force (USPSTF) members: Veterans Affairs Palo Alto Health Care System, Palo Alto, California (Owens); Stanford University, Stanford, California (Owens); Feinstein Institute for Medical Research, Northwell Health, Manhasset, New York (Davidson); Fairfax Family Practice Residency, Fairfax, Virginia (Krist); Virginia Commonwealth University, Richmond (Krist); Harvard Medical School, Boston, Massachusetts (Barry); University of California, San Francisco (Cabana); Oregon Health \& Science University, Portland (Caughey); Mayo Clinic, Rochester, Minnesota (Doubeni); Virginia Tech Carilion School of Medicine, Roanoke (Epling Jr); Temple University, Philadelphia, Pennsylvania (Kubik); University of Alabama at Birmingham (Landefeld); University of California, Los Angeles (Mangione); University of Massachusetts Medical School, Worcester (Pbert); Boston University, Boston, Massachusetts (Silverstein); Northwestern University, Evanston, Illinois (Simon); University of Hawaii, Honolulu (Tseng); Pacific Health Research and Education Institute, Honolulu, Hawaii (Tseng); Tufts University School of Medicine, Boston, Massachusetts (Wong)

Author Contributions: Dr Owens had full access to all of the data in the study and takes responsibility for the integrity of the data and the accuracy of the data analysis. The USPSTF members contributed equally to the recommendation statement.

Conflict of Interest Disclosures: Authors followed the policy regarding conflicts of interest described at https://www.uspreventiveservicestaskforce.org/ Page/Name/conflict-of-interest-disclosures. All members of the USPSTF receive travel reimbursement and an honorarium for participating in USPSTF meetings. Dr Barry reported receiving grants and personal fees from Healthwise.

Funding/Support: The USPSTF is an independent, voluntary body. The US Congress mandates that the Agency for Healthcare Research and Quality (AHRQ) support the operations of the USPSTF.
Role of the Funder/Sponsor: AHRQ staff assisted in the following: development and review of the research plan, commission of the systematic evidence review from an Evidence-based Practice Center, coordination of expert review and public comment of the draft evidence report and draft recommendation statement, and the writing and preparation of the final recommendation statement and its submission for publication. AHRQ staff had no role in the approval of the final recommendation statement or the decision to submit for publication.

Disclaimer: Recommendations made by the USPSTF are independent of the US government. They should not be construed as an official position of AHRQ or the US Department of Health and Human Services.

Additional Contributions: We thank Howard Tracer, MD (AHRQ), who contributed to the writing of the manuscript, and Lisa Nicolella, MA (AHRQ), who assisted with coordination and editing.

Additional Information: The USPSTF makes recommendations about the effectiveness of specific preventive care services for patients without obvious related signs or symptoms. It bases its recommendations on the evidence of both the benefits and harms of the service and an assessment of the balance. The USPSTF does not consider the costs of providing a service in this assessment. The USPSTF recognizes that clinical decisions involve more considerations than evidence alone. Clinicians should understand the evidence but individualize decision-making to the specific patient or situation. Similarly, the USPSTF notes that policy and coverage decisions involve considerations in addition to the evidence of clinical benefits and harms.

\section{REFERENCES}

1. American Psychiatric Association. Diagnostic and Statistical Manual of Mental Disorders. 5th ed. Washington, DC: American Psychiatric Association; 2013.

2. Langa KM, Larson EB, Crimmins EM, et al. A comparison of the prevalence of dementia in the United States in 2000 and 2012. JAMA Intern Med. 2017;177(1):51-58. doi:10.1001/jamainternmed.2016. 6807

3. Ward A, Tardiff S, Dye C, Arrighi HM. Rate of conversion from prodromal Alzheimer's disease to Alzheimer's dementia: a systematic review of the literature. Dement Geriatr Cogn Dis Extra. 2013;3(1): 320-332. doi:10.1159/000354370

4. Manly JJ, Tang MX, Schupf N, Stern Y, Vonsattel $J P$, Mayeux R. Frequency and course of mild cognitive impairment in a multiethnic community. Ann Neurol. 2008;63(4):494-506. doi:10.1002/ ana.21326
5. Larrieu S, Letenneur L, Orgogozo JM, et al. Incidence and outcome of mild cognitive impairment in a population-based prospective cohort. Neurology. 2002;59(10):1594-1599. doi:10 1212/01.WNL.0000034176.07159.F8

6. Roberts RO, Geda YE, Knopman DS, et al. The incidence of $\mathrm{MCl}$ differs by subtype and is higher in men: the Mayo Clinic Study of Aging. Neurology. 2012;78(5):342-351. doi:10.1212/WNL. Ob013e3182452862

7. Bischkopf J, Busse A, Angermeyer MC. Mild cognitive impairment-a review of prevalence, incidence and outcome according to current approaches. Acta Psychiatr Scand. 2002;106(6): 403-414. doi:10.1034/j.1600-0447.2002.01417.x

8. Ward A, Arrighi HM, Michels S, Cedarbaum JM Mild cognitive impairment: disparity of incidence and prevalence estimates. Alzheimers Dement. 2012;8(1):14-21. doi:10.1016/j.jalz.2011.01.002

9. Holsinger T, Deveau J, Boustani M, Williams JW Jr. Does this patient have dementia? JAMA. 2007;297(21):2391-2404. doi:10.1001/jama. 297.21.2391

10. Plassman BL, Williams JW Jr, Burke JR, Holsinger T, Benjamin S. Systematic review: factors associated with risk for and possible prevention of cognitive decline in later life. Ann Intern Med. 2010; 153(3):182-193. doi:10.7326/0003-4819-153-3201008030-00258

11. Ritchie K, Carrière I, Ritchie CW, Berr C, Artero S Ancelin ML. Designing prevention programmes to reduce incidence of dementia: prospective cohort study of modifiable risk factors. BMJ. 2010;341: c3885. doi:10.1136/bmj.c3885

12. Livingston $G$, Sommerlad A, Orgeta V, et al Dementia prevention, intervention, and care. Lancet. 2017;390(10113):2673-2734. doi:10.1016/S01406736(17)31363-6

13. Boyle PA, Buchman AS, Wilson RS, Leurgans SE, Bennett DA. Physical frailty is associated with incident mild cognitive impairment in community-based older persons. J Am Geriatr Soc. 2010;58(2):248-255. doi:10.1111/j.1532-5415.2009. 02671.x

14. Cooper C, Sommerlad A, Lyketsos CG, Livingston $\mathrm{G}$. Modifiable predictors of dementia in mild cognitive impairment: a systematic review and meta-analysis. Am J Psychiatry. 2015;172(4):323-334. doi:10.1176/appi.ajp.2014.14070878

15. Mukamal KJ, Kuller LH, Fitzpatrick AL, Longstreth WT Jr, Mittleman MA, Siscovick DS. Prospective study of alcohol consumption and risk of dementia in older adults. JAMA. 2003;289(11): 1405-1413. doi:10.1001/jama.289.11.1405 
16. Patnode CD, Perdue LA, Rossom RC, et al. Screening for Cognitive Impairment in Older Adults: An Evidence Update for the U.S. Preventive Services Task Force. Evidence Synthesis No. 189. Rockville, MD: Agency for Healthcare Research and Quality; 2019. AHRQ publication 19-05257-EF-1.

17. Patnode CD, Perdue LA, Rossom RC, et al. Screening for cognitive impairment in older adults: updated evidence report and systematic review for the US Preventive Services Task Force [published February 25, 2020]. JAMA. doi:10.1001/jama.2019. 22258

18. Luck T, Roehr S, Rodriguez FS, et al. Memory-related subjective cognitive symptoms in the adult population: prevalence and associated factors-results of the LIFE-Adult-Study. BMC Psychol. 2018;6(1):23. doi:10.1186/s40359-018-0236-1

19. Ginó S, Mendes T, Maroco J, et al. Memory complaints are frequent but qualitatively different in young and elderly healthy people. Gerontology. 2010;56(3):272-277. doi:10.1159/000240048

20. Stites SD, Karlawish J, Harkins K, Rubright JD Wolk D. Awareness of mild cognitive impairment and mild Alzheimer's disease dementia diagnoses associated with lower self-ratings of quality of life in older adults. J Gerontol B Psychol Sci Soc Sci. 2017 72(6):974-985. doi:10.1093/geronb/gbx100

21. Mormont E, Jamart J, Jacques D. Symptoms of depression and anxiety after the disclosure of the diagnosis of Alzheimer disease. J Geriatr Psychiatry Neurol. 2014;27(4):231-236. doi:10.1177/ 0891988714532021

22. Mate KE, Pond CD, Magin PJ, Goode SM, McElduff P, Stocks NP. Diagnosis and disclosure of a memory problem is associated with quality of life in community based older Australians with dementia. Int Psychogeriatr. 2012;24(12):1962-1971. doi:10. 1017/S1041610212001111

23. Chodosh J, Petitti DB, Elliott M, et al. Physician recognition of cognitive impairment: evaluating the need for improvement. J Am Geriatr Soc. 2004;52 (7):1051-1059. doi:10.1111/j.1532-5415.2004.52301.x

24. Olafsdóttir M, Skoog I, Marcusson J. Detection of dementia in primary care: the Linköping study. Dement Geriatr Cogn Disord. 2000;11(4):223-229. doi:10.1159/000017241

25. Valcour VG, Masaki KH, Curb JD, Blanchette PL. The detection of dementia in the primary care setting. Arch Intern Med. 2000;160(19):2964-2968. doi:10.1001/archinte.160.19.2964
26. Center for Medicare \& Medicaid Services (CMS). Annual wellness visit. CMS website. https://www.cms.gov/Outreach-and-Education/ Medicare-Learning-Network-MLN/MLNProducts/ Downloads/AWV_Chart_ICN905706.pdf. Published August 2018. Accessed January 6, 2020.

27. National Institute on Aging website. https://www.nia.nih.gov/. Accessed January 6, 2020.

28. Siu AL; U.S. Preventive Services Task Force. Behavioral and pharmacotherapy interventions for tobacco smoking cessation in adults, including pregnant women: U.S. Preventive Services Task Force recommendation statement. Ann Intern Med. 2015;163(8):622-634. doi:10.7326/M15-2023

29. Curry SJ, Krist AH, Owens DK, et al; US Preventive Services Task Force. Screening and behavioral counseling interventions to reduce unhealthy alcohol use in adolescents and adults: US Preventive Services Task Force recommendation statement. JAMA. 2018;320(18): 1899-1909. doi:10.1001/jama.2018.16789

30. LeFevre ML; U.S. Preventive Services Task Force. Behavioral counseling to promote a healthful diet and physical activity for cardiovascular disease prevention in adults with cardiovascular risk factors: U.S. Preventive Services Task Force recommendation statement. Ann Intern Med. 2014; 161(8):587-593. doi:10.7326/M14-1796

31. Grossman DC, Bibbins-Domingo K, Curry SJ, et al; US Preventive Services Task Force. Behavioral counseling to promote a healthful diet and physical activity for cardiovascular disease prevention in adults without cardiovascular risk factors: US Preventive Services Task Force recommendation statement. JAMA. 2017;318(2):167-174. doi:10.1001/ jama.2017.7171

32. Bibbins-Domingo K, Grossman DC, Curry SJ, et al; US Preventive Services Task Force. Statin use for the primary prevention of cardiovascular disease in adults: US Preventive Services Task Force recommendation statement. JAMA. 2016;316(19): 1997-2007. doi:10.1001/jama.2016.15450

33. Siu AL; U.S. Preventive Services Task Force. Screening for high blood pressure in adults: U.S. Preventive Services Task Force recommendation statement. Ann Intern Med. 2015;163(10):778-786. doi:10.7326/M15-2223

34. Siu AL; U S Preventive Services Task Force. Screening for abnormal blood glucose and type 2 diabetes mellitus: U.S. Preventive Services Task
Force recommendation statement. Ann Intern Med. 2015;163(11):861-868. doi:10.7326/M15-2345

35. Siu AL, Bibbins-Domingo K, Grossman DC, et al; US Preventive Services Task Force (USPSTF). Screening for depression in adults: US Preventive Services Task Force recommendation statement. JAMA. 2016;315(4):380-387. doi:10.1001/jama.2015. 18392

36. Moyer VA; U.S. Preventive Services Task Force. Screening for cognitive impairment in older adults: U.S. Preventive Services Task Force recommendation statement. Ann Intern Med. 2014 160(11):791-797. doi:10.7326/M14-0496

37. Fowler NR, Perkins AJ, Gao S, Sachs GA, Boustani MA. Risks and benefits of screening for dementia in primary care: the Indiana University Cognitive Health Outcomes Investigation of the Comparative Effectiveness of Dementia Screening (IU CHOICE) trial [published online December 2 , 2019]. J Am Geriatr Soc. 2019. doi:10.1111/jgs.16247

38. Raina $P$, Santaguida $P$, Ismaila $A$, et al. Effectiveness of cholinesterase inhibitors and memantine for treating dementia: evidence review for a clinical practice guideline. Ann Intern Med. 2008;148(5):379-397. doi:10.7326/0003-4819-148 5-200803040-00009

39. Ducharme FC, Lévesque LL, Lachance LM، et al. "Learning to become a family caregiver"-efficacy of an intervention program for caregivers following diagnosis of dementia in a relative. Gerontologist. 2011;51(4):484-494. doi:10. 1093/geront/gnr014

40. Samus QM, Johnston D, Black BS, et al. A multidimensional home-based care coordination intervention for elders with memory disorders: the maximizing independence at home (MIND) pilot randomized trial. Am J Geriatr Psychiatry. 2014;22 (4):398-414. doi:10.1016/j.jagp.2013.12.175

41. American Academy of Family Physicians (AAFP). Clinical preventive service recommendation: dementia. AAFP website. https://www.aafp.org/patient-care/clinicalrecommendations/all/dementia.html. Accessed January 6, 2020.

42. American Academy of Neurology (AAN) Practice guideline update: mild cognitive impairment. AAN website. https://www.aan.com/ Guidelines/home/GetGuidelineContent/882. Published 2017. Accessed January 6, 2020. 